\title{
Efficacy of rituximab in non-paraneoplastic autoimmune retinopathy
}

\author{
Katherine Boudreault ${ }^{1,2,3}$, Sally Justus ${ }^{2,3}$, Jesse D. Sengillo ${ }^{2,3,4}$, Kaspar Schuerch ${ }^{2,3}$, Winston Lee ${ }^{2,3}$, \\ Thiago Cabral ${ }^{2,3,5,6}$ and Stephen H. Tsang ${ }^{2,3,7^{*}}$
}

\begin{abstract}
Background: Autoimmune retinopathy (AIR) is a rare but potentially blinding condition that is often underdiagnosed. Common features in AIR presentation include rapidly progressive vision loss with abnormal electrophysiological responses of the retina associated with positive anti-retinal antibodies. AIR is also challenging to treat, and thus, the introduction of new potential therapeutic agents is welcomed. The goal of this communication is to assess the effects of rituximab infusions on electroretinogram (ERG) responses and visual function outcomes in patients with non-paraneoplastic autoimmune retinopathy (nPAIR).
\end{abstract}

Results: Following infusion(s), three out of five patients showed no evidence of disease progression or improved, while two patients continued to progress on ERG. One patient demonstrated improvement in visual acuity (2 lines) in both eyes. ERG responses provided objective monitoring of patients' visual function and response to immunosuppression over time.

Conclusions: These findings suggest that patients with npAIR unresponsive to other immunosuppression therapies may benefit from rituximab infusion, although stabilization rather than improvement was more frequently the outcome in our case series. Furthermore, regularly scheduled ERG follow-up examinations are recommended for monitoring patients' progression during treatment.

Keywords: Autoimmune retinopathy, Rituximab, Treatment, Multi-modal imaging, Electroretinography

\section{Background}

Autoimmune retinopathies (AIR) comprise a spectrum of relatively uncommon autoimmune retinal diseases. Although AIR have been studied for the past 40 years [1, 2], they remain difficult to diagnose [3] and treat. AIR include such conditions as paraneoplastic autoimmune retinopathy (pAIR), which can be further subdivided into cancerassociated retinopathy (CAR) and melanoma-associated retinopathy (MAR). In the absence of malignancy, the condition is referred to as non-paraneoplastic autoimmune retinopathy (npAIR). A commonality uniting pAIR and npAIR is that in both conditions, the integrity and function of various retinal cells, including cones, rods, and bipolar cells, are affected by antiretinal antibodies (ARAs) that are believed to arise from molecular mimicry [4]. The cell types that are

\footnotetext{
* Correspondence: phosphodiesterase.gamma@gmail.com

Jonas Children's Vision Care, and Bernard \& Shirlee Brown Glaucoma Laboratory, Columbia University Medical Center, New York, NY, USA

${ }^{3}$ Department of Ophthalmology, Columbia University, New York, NY, USA

Full list of author information is available at the end of the article
}

most affected in each patient, and thus the initial signs and symptoms, likely depend on which retinal proteins are targeted by the ARAs [5-7]. Consequently, this causes heterogeneity in clinical presentation among patients, including central vision loss, variable changes in visual field, retinal structure, and morphology [8]. Recently, a panel of experts proposed a list of key diagnostic criteria for AIR, among which included: the absence of an apparent cause for visual dysfunction, an abnormal ERG, and the presence of serum ARAs [9]. Until now, there is no standard therapy or established treatment protocol, and patient outcomes following intervention are variable. However, a drug called rituximab has garnered interest as a potential treatment option.

Rituximab is a monoclonal antibody that binds to $\mathrm{CD} 20$, a non-glycosylated protein expressed on the surface of B lymphocytes (B-cells), inducing B-cell lysis [10]. It was first approved by the FDA for the treatment of B-cell lymphoma, although recently it has been applied to a variety of autoimmune disorders [11]. However, its use has not been extensively explored for 
immune-related retinal conditions, and only case reports and one case series have discussed rituximab administration for patients with AIR [12-19]. Here, we present a case series of five patients exploring the effects of rituximab therapy for the treatment of npAIR as assessed by electrodiagnostic testing. Our aim is to provide a reference for clinicians who are seeking new options for managing this complicated disease and to demonstrate the utility of the ERG as a means of assessing response to immunosuppression in npAIR.

\section{Methods}

We performed a retrospective review of all cases of npAIR diagnosed at the Edward S Harkness Eye Institute at New York-Presbyterian Hospital (NYPH) between 2009 and 2016. Five cases were selected based on the following inclusion criteria: (1) they received at least one rituximab infusion during their disease course, and (2) they had a minimum of a six-month follow-up to assess visual function with electrodiagnostic testing, visual acuity, and multimodal imaging, as well as visual field testing when available. The diagnosis of npAIR was based on peer-reviewed diagnostic criteria [9].

Detection of ARAs in all patients was confirmed by one of two laboratories: The Ocular Immunology Laboratory located at Oregon Health \& Science University (Portland, Oregon) or The University of California at Davis Laboratory (Davis, California). Autoantibody detection was performed as previously described $[20,21]$. Briefly, serum was collected from patients and the presence of anti-retinal antibodies was determined by western blot analysis. Western blot band thickness was compared between tests and used to assess the change in response for specific antibodies over time.

Full-field electroretinograms (ffERGs) (Diagnosys LLC, Lowell, Massachusetts, USA) were recorded from both eyes with DTL electrodes according to the standards from the International Society for Clinical Electrophysiology of Vision (ISCEV) [22] in both scotopic and photopic states. When $30 \mathrm{~Hz}$ flicker amplitudes were lower than 5 microvolts $(\mu \mathrm{V})$, BurianAllen contact lens electrodes were used to record the electric responses. The amplitudes and implicit times obtained from both eyes of each patient were compared with age-matched normal values, in which the boundary of normal limits represented two standard deviations from the mean.

The main outcome was the effect of rituximab on visual function before and after treatment and was determined based on two independent criteria: (1) stability or improvement in ERG scotopic and/or photopic response, using the last response before treatment with rituximab as the baseline (ratio $=1$ ); and (2) improvement or stability in best corrected visual acuity (BCVA), using the last visual acuity measurement before initiation of treatment with rituximab as the baseline (ratio = 1). Ratios were calculated for ERG and BCVA by comparing the post-treatment response to baseline response $\left(\frac{\text { after rituximab }}{\text { just before rituximab }}\right)$, such that values greater than 1 indicate improvements in visual function, and values less than one indicate declines in function. Secondary outcomes included subjective stability or improvement on visual field testing; retinal structure, as assessed by spectraldomain optical coherence tomography (SD-OCT) when available; and assessment of antibody titer after rituximab infusions. The following data were retrieved retrospectively for each patient: demographics (age, gender); medical history (history of other autoimmune diseases); clinical features; non-rituximab treatment data (treatment(s), response); and rituximab treatment data (dose, adverse reactions, response).

\section{Results}

\section{Demographics}

In the present study, patients ranged between 10 and 70 years of age; one was an African-American male, and four patients were Caucasian females. Two patients were originally diagnosed with suspected inherited retinal dystrophies, but in both cases, visual acuity and/or visual field loss deteriorated rapidly, which is not consistent with the typically slow natural history of inherited retinal dystrophies. The median follow-up period was 51 months. Three patients (P1, 2, and 5) had a past medical history of systemic autoimmune disorder, and $\mathrm{P} 4$ was diagnosed with Crohn disease 2 years after the initial npAIR diagnosis (Table 1). Four were on other immunosuppressants prior to rituximab, with an initially positive response to treatment in three patients that eventually failed, prompting initiation of rituximab. Four patients presented with rodcone dysfunction, and one patient (P4) presented with cone-rod dysfunction on electroretinography (Fig. 1).

\section{Response to rituximab across patients}

Median follow up period after first rituximab infusion was 15 months (Table 2). The rituximab treatment regiment varied between patients with regards to the number of infusions, dosage, and interval between doses, which was adjusted based on patients' B cell count and specialist preferences. After rituximab infusions, patients 1 and 5's flicker ratio stabilized and P4's improved, while patients' 2 and 3 flicker and/or rod ratios showed a rapid decline below a ratio of 1 . The best-corrected visual acuity ratio correlated closely with ERG flicker ratio in most cases, except for P1, who developed a cataract in one eye. Only one patient (P4) demonstrated improvement in visual acuity (2 lines with Snellen) in both eyes 
Table 1 Clinical characteristics of npAIR patients

\begin{tabular}{|c|c|c|c|c|c|c|c|}
\hline $\begin{array}{l}\text { Case\#/Sex/Age } \\
\text { (y) }\end{array}$ & $\begin{array}{l}\text { Symptoms onset } \\
\text { before confirmed } \\
d x(m)\end{array}$ & $\begin{array}{l}\text { Other autoimmune } \\
\text { disorder(s) }\end{array}$ & $\begin{array}{l}\text { Total Follow-up } \\
\text { duration }(y, m)\end{array}$ & $\begin{array}{l}\text { WB bands at } \\
\text { presentation } \\
(\mathrm{kDa})\end{array}$ & $\mathrm{npAIR} / \mathrm{RP}$ & $\begin{array}{l}\text { BCVA OS/OD } \\
\text { at presentation }\end{array}$ & $\begin{array}{l}\text { Immunosuppressors before } \\
\text { rituximab/Response before } \\
\text { failure }\end{array}$ \\
\hline $1 / F / 61$ & 24 & Psoriasis, HypoTSH & $6 y 9 m$ & $\begin{array}{l}33,45,55 \\
64,72,90\end{array}$ & Yes/No & $\begin{array}{l}20 / 150 \\
20 / 150\end{array}$ & $\begin{array}{l}\text { MMF, I/Improved and } \\
\text { stabilized } \times 60 \text { months on } R\end{array}$ \\
\hline 2/M/65 & 28 & Cutaneous LE & $4 y 3 m$ & $\begin{array}{l}40,46 \\
\text { (enolase), } 68\end{array}$ & Yes/Yes & $\begin{array}{l}20 / 40 \\
20 / 40\end{array}$ & $\begin{array}{l}\text { MMF/No response after } \\
\text { 7-month trial }\end{array}$ \\
\hline $3 / F / 16$ & 36 & None & $2 \mathrm{y} 10 \mathrm{~m}$ & $\begin{array}{l}23 \text { (HSP27), } \\
28,34,36,39, \\
46 \text { (enolase), } \\
62\end{array}$ & Yes/Yes & $\begin{array}{l}20 / 40 \\
20 / 40\end{array}$ & None \\
\hline 4/F/10 & 12 & Crohn Disease & $2 y 2 m$ & 28,92 & Yes/No & $\begin{array}{l}20 / 150 \\
20 / 125\end{array}$ & $\begin{array}{l}\text { C, P, MMF, IVIG/Improved } \times \\
5 \text { months }\end{array}$ \\
\hline $5 / F / 70$ & 6 & $\begin{array}{l}\text { Hashimoto } \\
\text { Thyroiditis, Sjogren } \\
\text { syndrome }\end{array}$ & $4 y 6 m$ & 42 & Yes/No & $\begin{array}{l}20 / 25 \\
20 / 30\end{array}$ & $\begin{array}{l}\text { MMF, P/Initial improvement, } \\
\text { than compliance variable with } \\
\text { stepwise decrease in visual } \\
\text { function }\end{array}$ \\
\hline
\end{tabular}

$Y$ years, $m$ months, $k D a$ kilodalton, WB western blot, BCVA best corrected visual acuity, HypoTSH hypothyroidism, Cutaneous LE Cutaneous Lupus Erythematosus, $M M F$ mycophenolate mofetil, I infliximab, $R$ rituximab, $C$ cyclosporine, $P$ prednisone, IVIG intravenous immunoglobulins

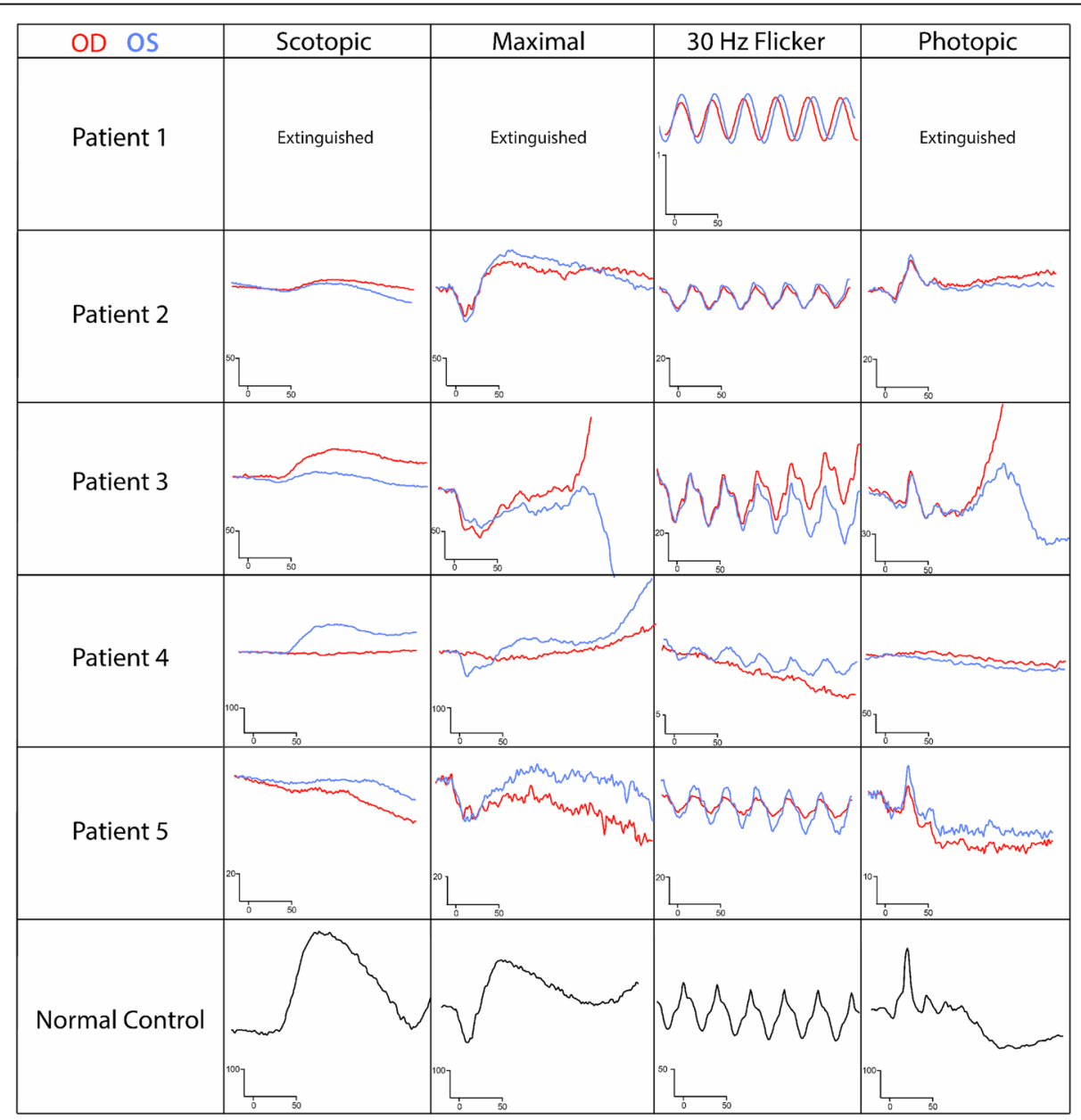

Fig. 1 Scotopic and photopic ERG curves at presentation for each patient. Patient 1 (P1) ERG values are presented in the top row, and each patient's data sequentially follows, with a normal control provided on the bottom row. Scales vary between patients. OD shown in red, OS in blue 
Boudreault et al. Orphanet Journal of Rare Diseases (2017) 12:129

Page 4 of 15

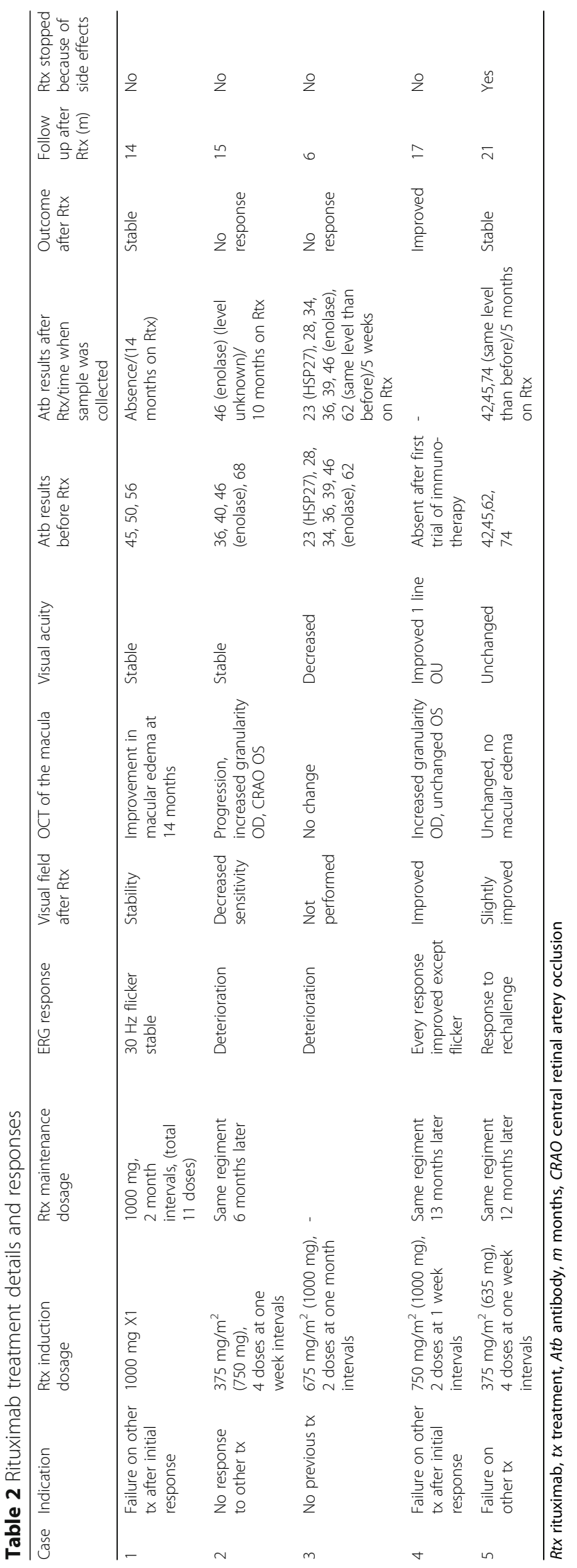


(Fig. 2). One patient (P5) developed more frequent sinus infections, nodular scleritis, and zoster ophthalmicus during the course of her treatment. Table 3 summarizes patient outcomes.

\section{Cases}

\section{Patient 1 (P1)}

A 61 year-old woman complained of rapid, progressive vision loss. On September 28th, 2009, her vision was recorded at 20/150 bilaterally. The fundoscopic examination revealed extensive mottling of the retinal pigment epithelium (RPE) and limited pigment migration only in the left eye (Fig. 3a). Immunoblot analysis showed reactivity against $33,45,55,64,72$, and $90-\mathrm{kDa}$ proteins, while work-up for neoplastic and infectious causes was negative. Mycophenolate mofetil was initiated, than replaced by infliximab at a dose of $400 \mathrm{mg}$ once every 2 months. At the end of 2011, the first ffERG performed showed an extinguished rod response, and $30 \mathrm{~Hz}$ flicker response was approximately $0.3 \mu \mathrm{V}$ bilaterally (Fig. 1a). Her vision progressively improved to $20 / 25$ in the right eye and 20/30 in the left eye. The ERG showed progressive improvement on $30 \mathrm{~Hz}$ flicker response (Fig. 2a). However, around September 2014, her ERG responses deteriorated, she developed macular edema bilaterally,

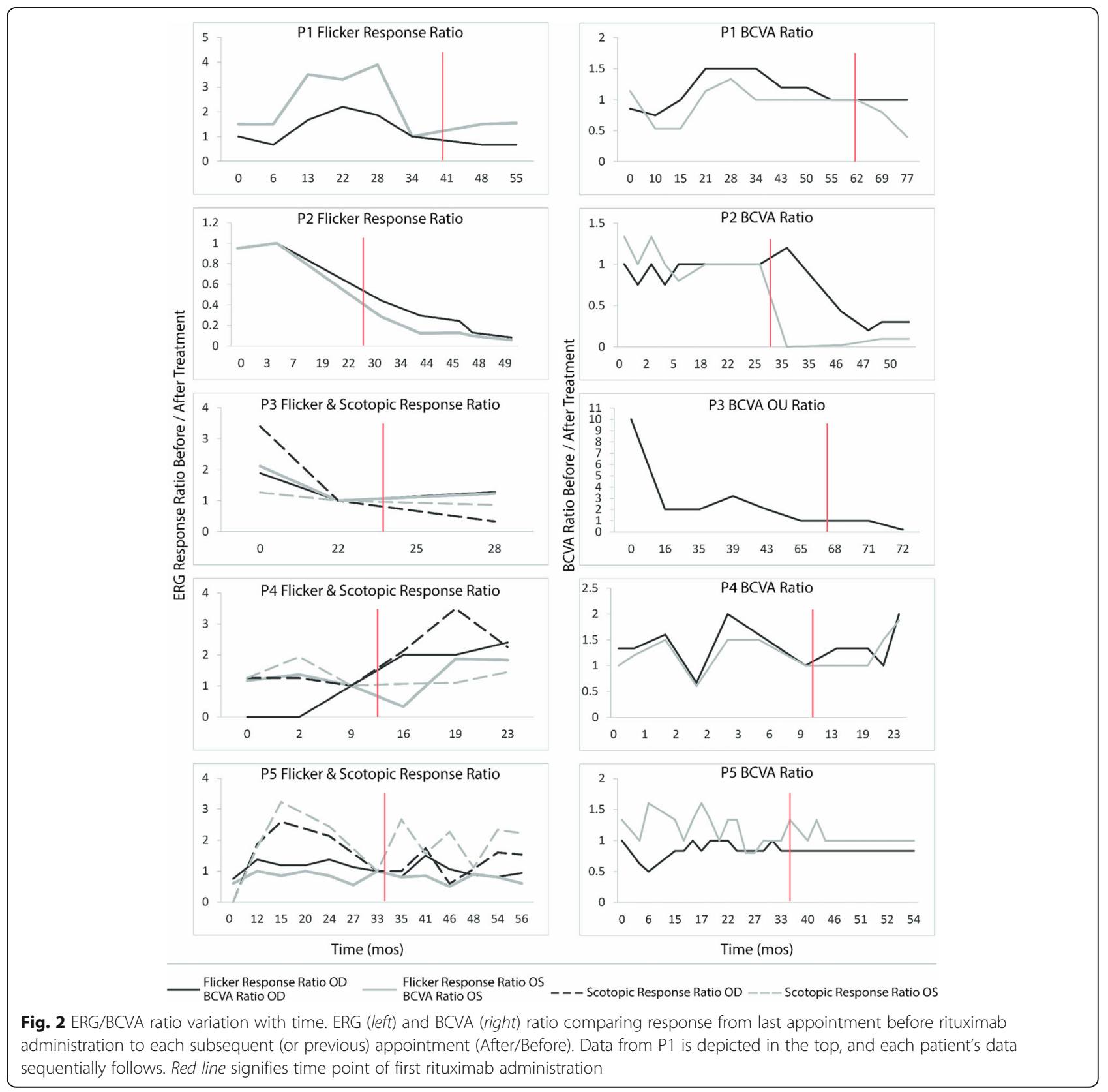


Table 3 Synopsis of patient outcomes

\begin{tabular}{llllll}
\hline & P1 & P2 & P3 & P4 & P5 \\
\hline BCVA & S & S & D & I & S \\
ERG & S & D & D & I & S \\
Visual fields & S & D & NA & I & I \\
ARA (\# of bands) & $3 \rightarrow 0$ & $3 \rightarrow 1$ & $7 \rightarrow 7$ & $0 \rightarrow 0$ & $4 \rightarrow 3$ \\
AE & - & - & - & - & 2 \\
Overall & Stable & Poor & Unresponsive & Positive & Stable
\end{tabular}

$S$ Stable, I Improved, D Deteriorated, NA not applicable, AE Adverse Events and her vision dropped. Immunoblot analysis showed reactivity against 45,50 , and $56-\mathrm{kDa}$ proteins. Infliximab was discontinued and rituximab infusions were initiated. Repeat immunoblot analysis showed no change 7 months after rituximab institution. In June 2016, her vision had slightly decreased in the left eye, but this was attributed to a posterior subcapsular cataract. Macular edema was slightly improved bilaterally when compared to SD-OCT images taken prior to initiation of rituximab (Fig. 3b). Her visual field and ERG responses have remained within the same range since (Fig. 3c). Fourteen months after institution of rituximab, repeat western blot analysis showed
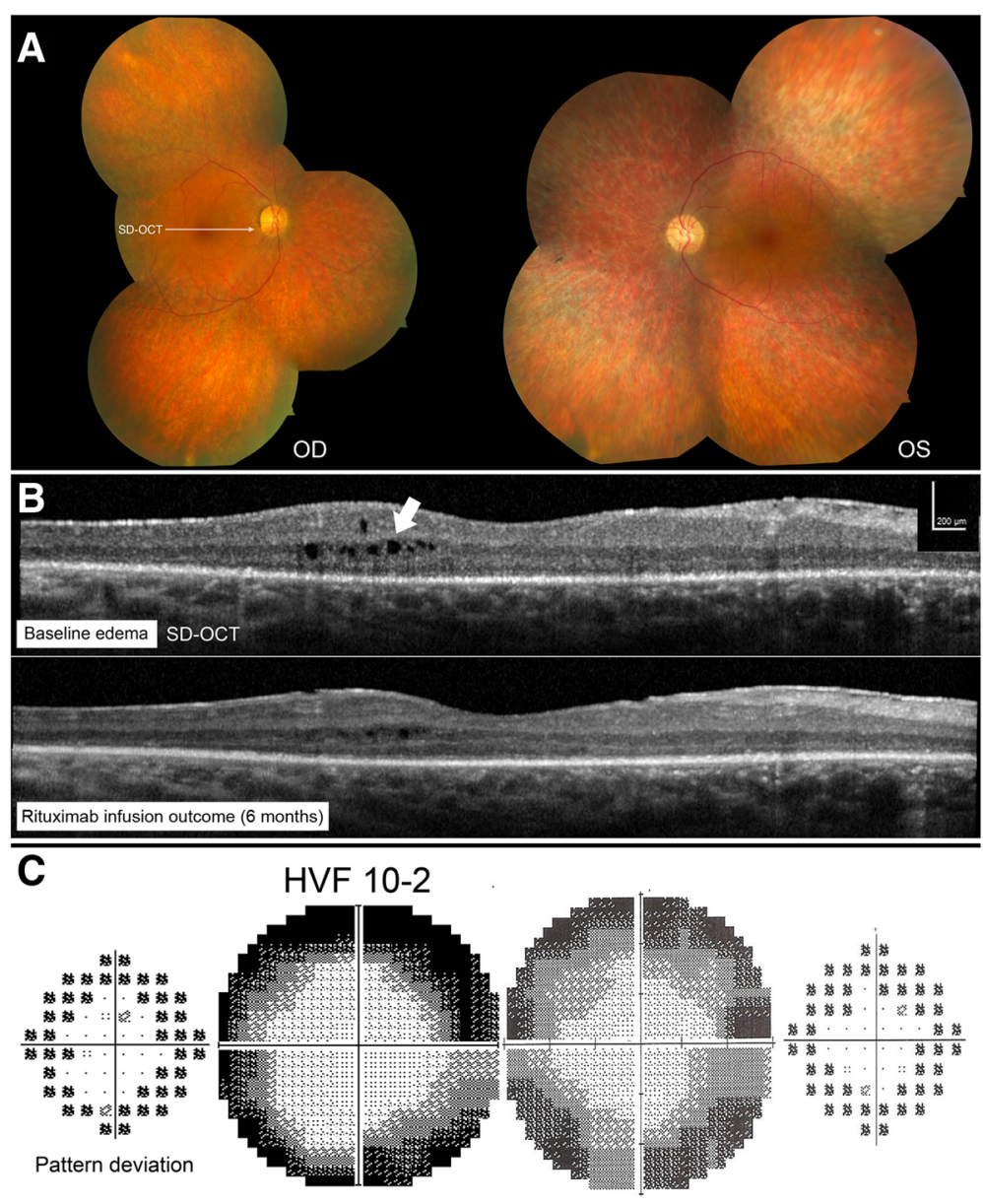

D Anti-Retinal Antibody Analysis

$$
v_{0}, 50
$$
$\hat{\beta} \hat{\jmath}$ $\vartheta$

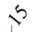

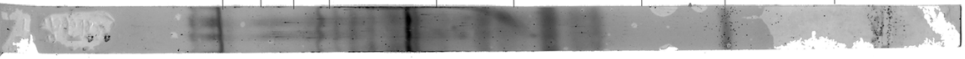

Fig. 3 P1 imaging and functional assessments. P1 fundus picture OU at presentation (a). OCT line OD showed new cystic macular edema, which improved after rituximab infusions (b). Humphrey visual field (HVF) 10-2 OD grey scale and pattern standard deviation showed diffuse peripheral loss while on infliximab (2012, left), which stabilized after a few months on rituximab infusions (2015, right) (c). Western blot analysis after 7 months (top) and 14 months (bottom) on rituximab showed almost complete absence of reaction at follow-up (d) 
undetectable antibody levels (Fig. 3d). For these reasons, she was declared stable on rituximab.

\section{Patient 2 (P2)}

A 65 year-old man reported progressive peripheral vision loss. Initial visual acuity was $20 / 25$ bilaterally. At his first visit in April 2012, his visual acuity dropped to 20/40 bilaterally. Fundus imaging showed myopic fundi, attenuated vessels and mottling of the retina (Fig. 4a). OCT showed a lamellar hole with cysts in the right eye. ffERG showed severe rod-cone dysfunction (Fig. 1b). He was diagnosed with late-onset retinitis pigmentosa. However, in 2013, there was a significantly decreased response on visual field testing. ARA testing was positive for anti$40 \mathrm{kDa}$, anti-46 kDa (enolase), and anti-68 kDa proteins.
Work-up for malignancy was negative, and ERG responses continued to deteriorate (Fig. 2b). The patient was prescribed mycophenolate mofetil $1000 \mathrm{mg}$ twice a day, but seven months later, visual fields were worse and antibody testing, unchanged. The decision was made to escalate treatment and start rituximab infusions. Six months later, OCT of the macula showed a novel granular appearance at the level of the outer retinal layers (Fig. 4b), and the visual field showed continued deterioration in both eyes (Fig. 4c). The patient eventually developed left central vein occlusion and vision dropped to light perception in the left eye. Antibody testing showed reactivity against $46-\mathrm{kDa}$ proteins, and ERG testing comparing responses before starting immunosuppression showed tremendous progression (Fig. 2b). He
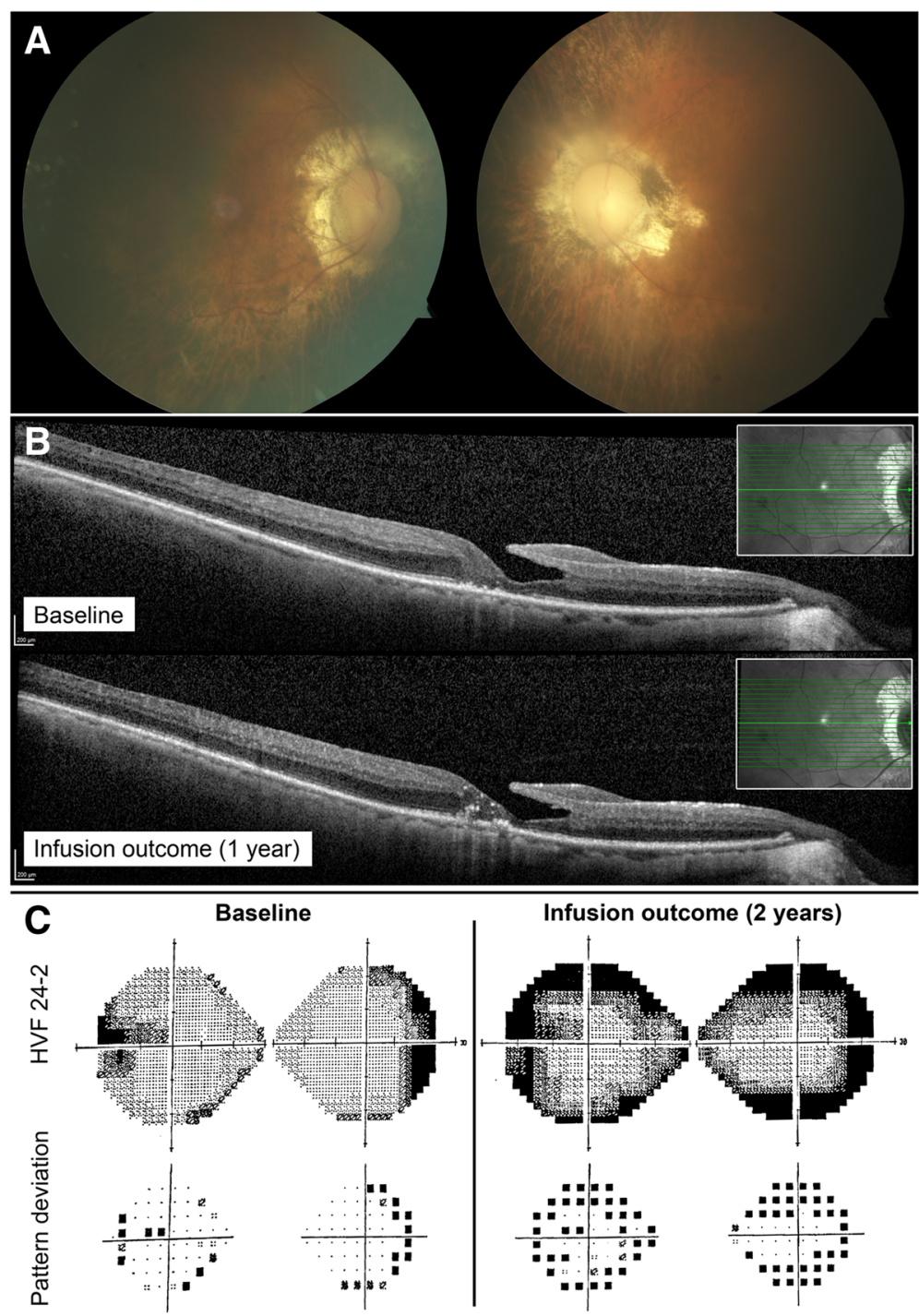

Fig. 4 P2 imaging and functional assessments. P2 fundus pictures OU (a). OCT line OD showing lamellar hole with cysts in the right eye before starting rituximab (2014, top), which continued to deteriorate on rituximab treatment (2015, bottom) (b). HVF 24-2 OU grey scale and pattern deviation before (2013, left) and after (2015, right) initiation of rituximab (c) 
eventually stabilized with plasmapheresis treatment. Thus, this patient was classified as having a poor outcome on rituximab.

\section{Patient 3 (P3)}

A 16 year-old girl without significant past medical history complained of bilateral rapid central visual loss associated with photopsia over the summer of 2010. There was no family history of autoimmune disorders or hereditary retinopathies. The visual loss deteriorated in a stepwise fashion, starting at $20 / 40$ in 2010 , to $20 / 200$ in 2011, and eventually to $20 / 400$ bilaterally in 2015 . The first dilated fundus examination at NYPH showed a bull's eye maculopathy (Fig. 5a) and thinning of the retinae with photoreceptor layer loss on OCT, mostly centrally. Goldmann visual fields demonstrated decreased sensitivity, particularly in the maculae in both eyes (Fig. 5c). ffERG showed decreased rod response more so than was observed for cones, with an electronegative appearance (Fig. 1c). In June 2015, testing for ARAs showed reactivity against 23 (HSP27), 28, 34, 36, 39, 46 (anti-enolase), and 62-kDa proteins. Malignancy work up was unremarkable. In the meantime, whole exome sequencing results returned positive for two deleterious mutations in the MSFD8 gene. Mutations in this gene usually cause neuronal ceroid lipofuscinose (NCL), a neurologic disorder that usually presents with early-onset epilepsy, retinal degeneration, and progressive mental and motor deterioration [23]. In the absence of systemic findings and the rarity of case reports of non-syndromic MSFD 8 patients with late-onset visual loss [24], it was entertained that the patient may have been genetically susceptible to developing vision loss. The combination of a genetic background predisposed towards retinal degeneration as well as numerous antiretinal antibodies and their proven pathogenicity (enolase and SHP27, especially) could have induced the rapid
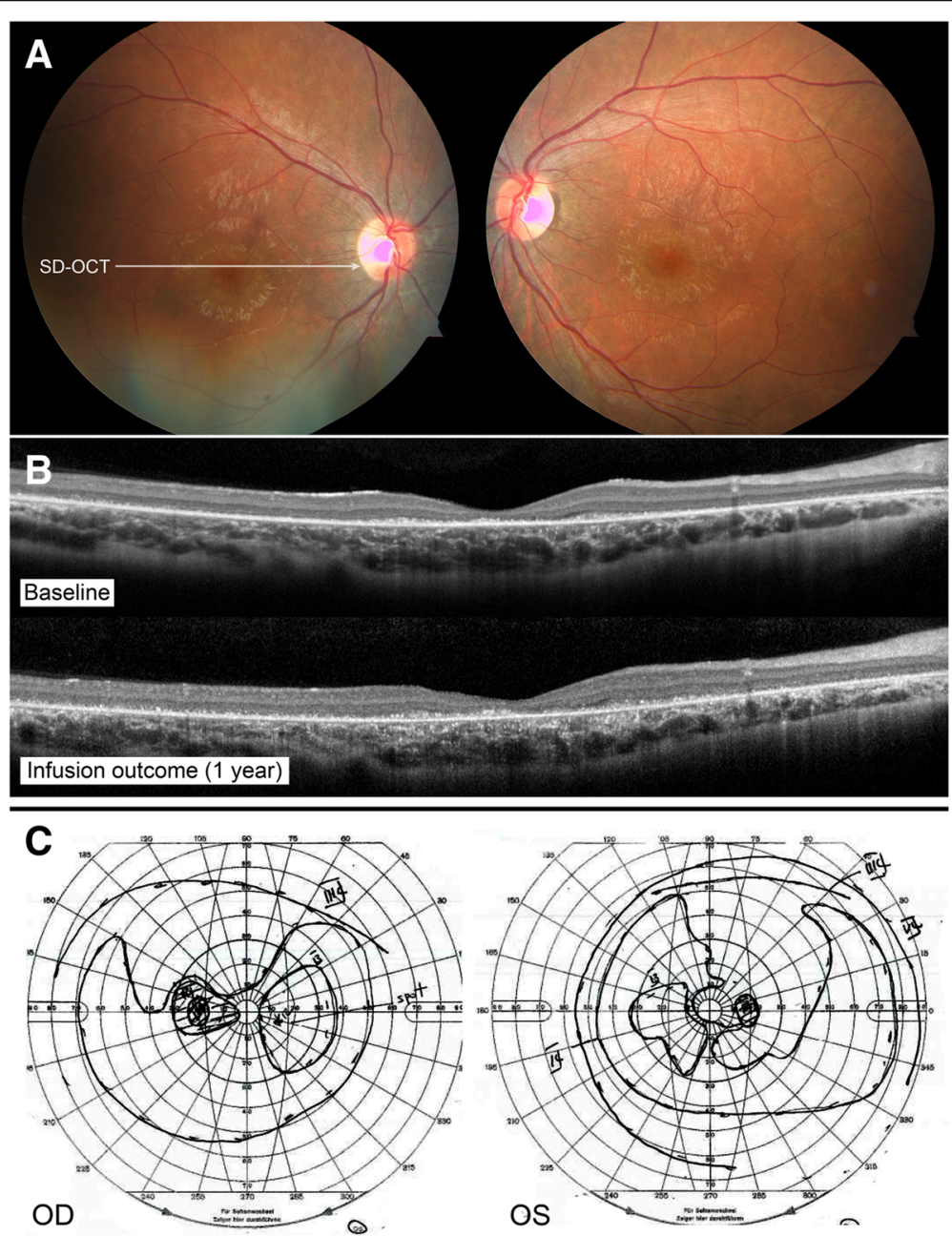

Fig. 5 P3 imaging and functional assessments. P3 fundus pictures OU (a). OCT line in OD showing retinal thinning and progression of granular deposits at the EZ line between spring 2015 and end of 2015, after rituximab infusion (top and bottom, respectively) (b). Goldmann visual fields in both eyes at first visit (c) 
vision deterioration observed in this patient. In August 2015, rituximab infusions were initiated. Repeated antibody testing five weeks after the first infusion was unchanged. Four months after the second infusion, there was stability in $30 \mathrm{~Hz}$ flicker response, but the rod response continued to progress on ERG testing. The vision deteriorated, and an OCT showed worsening granularity when comparing images before and after treatment (Fig. 5b). Thus, this patient was classified as non-responsive to rituximab.

\section{Patient 4 (P4)}

A 10 year-old girl without significant past medical history and normal ocular exams before symptom onset complained of decreased color vision with phosphene six months prior to visiting the Columbia Eye Institute. In June 2014, BCVA was recorded at 20/150 OD and 20/125 OS. Fundus examination revealed marked vascular attenuation and a large zone of macular RPE and choriocapillaris atrophy in the right eye. The left fundus showed only subtle chorioretinal atrophy centrally (Fig. 6a). OCT demonstrated
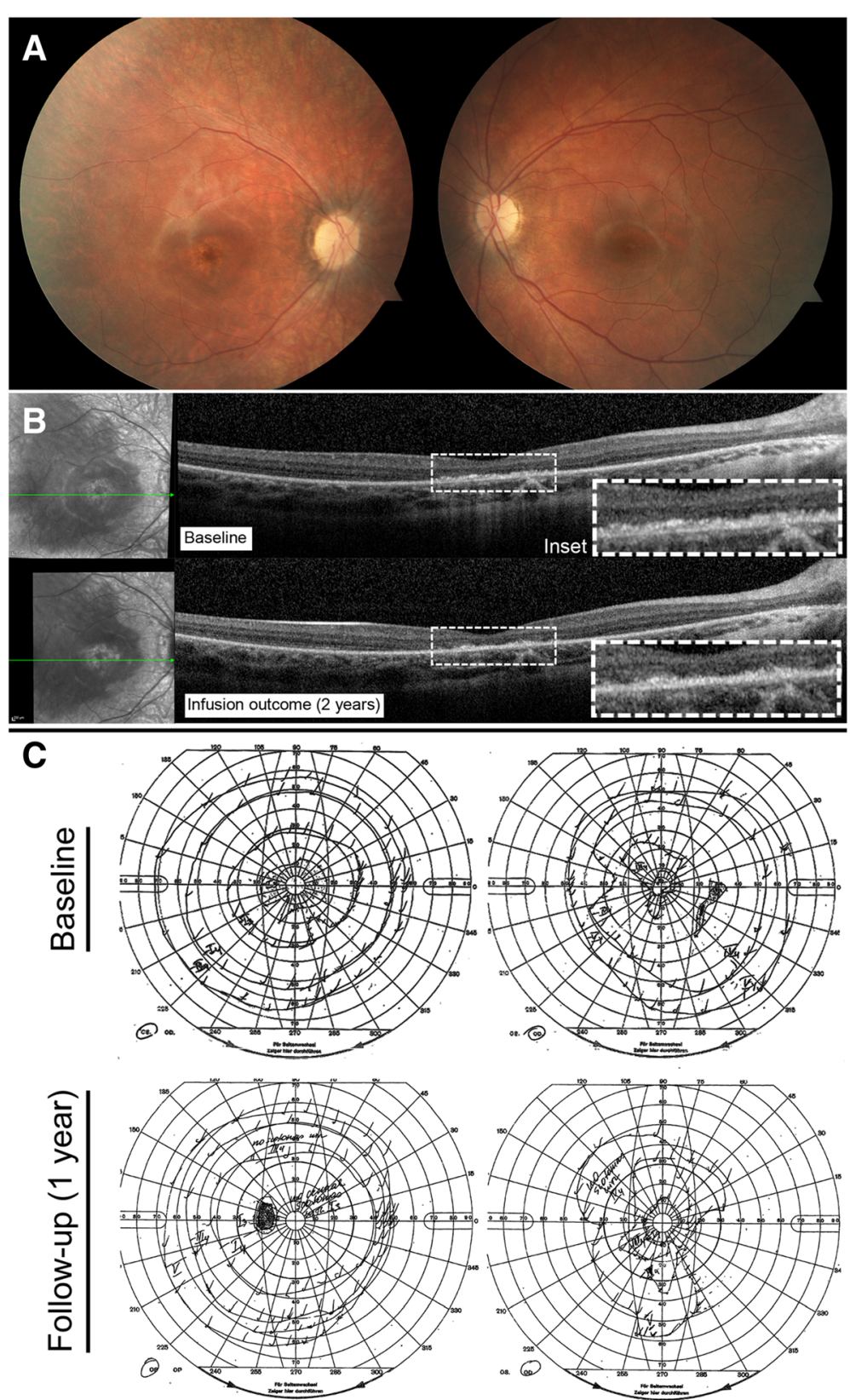

Fig. 6 P4 imaging and functional assessments. P4 fundus pictures OU (a). OCT line OD showing loss of EZ line centrally and granular deposition before starting rituximab (2014, top), which changed minimally on rituximab treatment (2016, bottom) (b). Goldmann visual field in both eyes before $(2014$, top) and after $(2015$, bottom) initiation of rituximab (c) 
thinning of the retina and loss of the ellipsoid zone (EZ) line centrally in the right eye as well as partial loss of the EZ line in the left eye. Goldmann visual field showed no response to stimuli smaller than II2 in the right eye and no response to stimuli smaller than I3 in the left eye. ffERG demonstrated asymmetry between both eyes, with a profound decrease in cone response bilaterally and residual rod response in the left eye (Fig. 1d). Full body work-up, serologies for infectious diseases, and genetic testing (retinal panel) returned negative. In June 2014, ARAs showed reactivity against $28-\mathrm{kDa}$ and $92-\mathrm{kDa}$ proteins. Vision further decreased to 20/300 OD and 20/250 OS, and the Goldmann visual field deteriorated. An immunologist initiated intravenous immunoglobulins (IVIg) $2 \mathrm{~g} / \mathrm{kg}$ with one dose of methylprednisolone at $1000 \mathrm{mg}$ IV, followed by maintenance treatment with prednisone $60 \mathrm{mg}$, mycophenolate mofetil $1000 \mathrm{mg}$ twice daily, and cyclosporine $150 \mathrm{mg}$ daily. A month later, vision returned to $20 / 100$ bilaterally, and antibody testing came back negative, so a second dose of IVIg was given. However, in January 2015, the visual field worsened again, and ERG responses dropped. Medication was discontinued and the patient received one dose of rituximab. Seven months later, all ERG responses showed improvement or stability except for $30 \mathrm{~Hz}$ flicker in the left eye, which declined slightly. Visual acuity improved in the right eye, while an OCT before and after rituximab infusion showed mildly increased granular appearance in the right eye and no change in the left (Fig. 6b). I4 stimulus was now seen centrally in the right eye, and the central scotoma disappeared in the left eye (Fig 6c). Her B-cell level dropped and remained within the appropriate range for 12 months, and she therefore received a second dose of rituximab 13 months later. Subsequently, there was a new response to I2 stimulus centrally in the left eye and to I3 in the right eye. Overall, after rituximab, vision improved from $20 / 200$ to $20 / 100$ in the right and from 20/150 to 20/80 in the left eye; ERG responses also improved bilaterally and stabilized. This patient was thus determined as showing improvement on rituximab.

\section{Patient 5 (P5)}

A 70 year-old woman with known numerous autoimmune disorders reported night blindness for a few years, but blind spots in both eyes caused her to seek care. In December 2011, her vision was recorded at 20/ 25 in the right and 20/30 in the left eye. Funduscopic examination revealed extensive mottling of the RPE in the periphery, with limited peri-vascular pigment migration and vessel attenuation (Fig. 7a). ffERG showed an extinguished rod response, electronegative maximal response, and $30 \mathrm{~Hz}$ flicker response amplitudes that were around $12 \mu \mathrm{V}$ bilaterally (Fig. 1e). Immunoblot analysis showed reactivity against $42-\mathrm{kDa}$ (arrestin) proteins, and a neoplastic and infectious work-up was negative. Visual field worsened, which prompted the initiation of mycophenolate mofetil $500 \mathrm{mg}$ twice daily eight months later. Rod-specific ERG responses improved initially, but eventually, visual field showed continued progression even with increased dosage of mycophenolate mofetil and the addition of oral prednisone. Repeated immunoblot analysis showed reactivity against multiple antigens, and repeated ERG showed continued deterioration in rod response. On September 2014, the patient's immunosuppression treatment was replaced with three rituximab infusions administered over a period of three months. ERG responses fluctuated but remained stable. However, because of recurrent sinus infections, a second trial of rituximab was initiated only one year later after recurrence of visual symptoms. The patient subsequently developed ophthalmic zoster and nodular scleritis in the left eye. This prompted concern for an immunological deficiency secondary to rituximab, although her immunoglobulin levels were within the normal range, which was reassuring. Overall, the ERG, OCT (Fig. 7b), visual fields (Fig. 7c), and visual acuity in the right eye remained stable with rituximab, and the patient continued the medication. Visual acuity was slightly decreased in the left eye because of a cataract. This patient was thus classified as stabilized on rituximab.

\section{Discussion}

AIR is a rare autoimmune disorder characterized by the production of ARAs that target retinal proteins. Normally when the body encounters a foreign pathogen, B cells bind to a unique antigen, which causes them to mature into antibody-producing plasma cells or memory B cells, which trigger an immune response. However, in autoimmune conditions such as AIR, the B cells become reactive to self-antigens [25] and begin to produce pathogenic ARAs. This process eventually induces retinal cell death and leads typically to a more rapid and progressive vision loss [2] compared to hereditary retinal degenerations, which show a slow mean decrease of $10 \%$ per year on $30 \mathrm{~Hz}$ flicker ERG response in patients with retinitis pigmentosa, for example [26].

In this case series, we exclusively studied patients with npAIR, which distinguishes itself from other forms of AIR by the lack of malignancy. In our cohort of npAIR patients, a high percentage (80\%) were female, which is typical of autoimmune conditions [27], and $80 \%$ had another coexisting autoimmune condition, which is also reported in patients with AIR according to previous studies [27]. All patients had abnormal ERG responses, rapid disease progression and positive testing for ARAs. Four patients were previously taking an alternative immunosuppressant, and three initially responded well, 

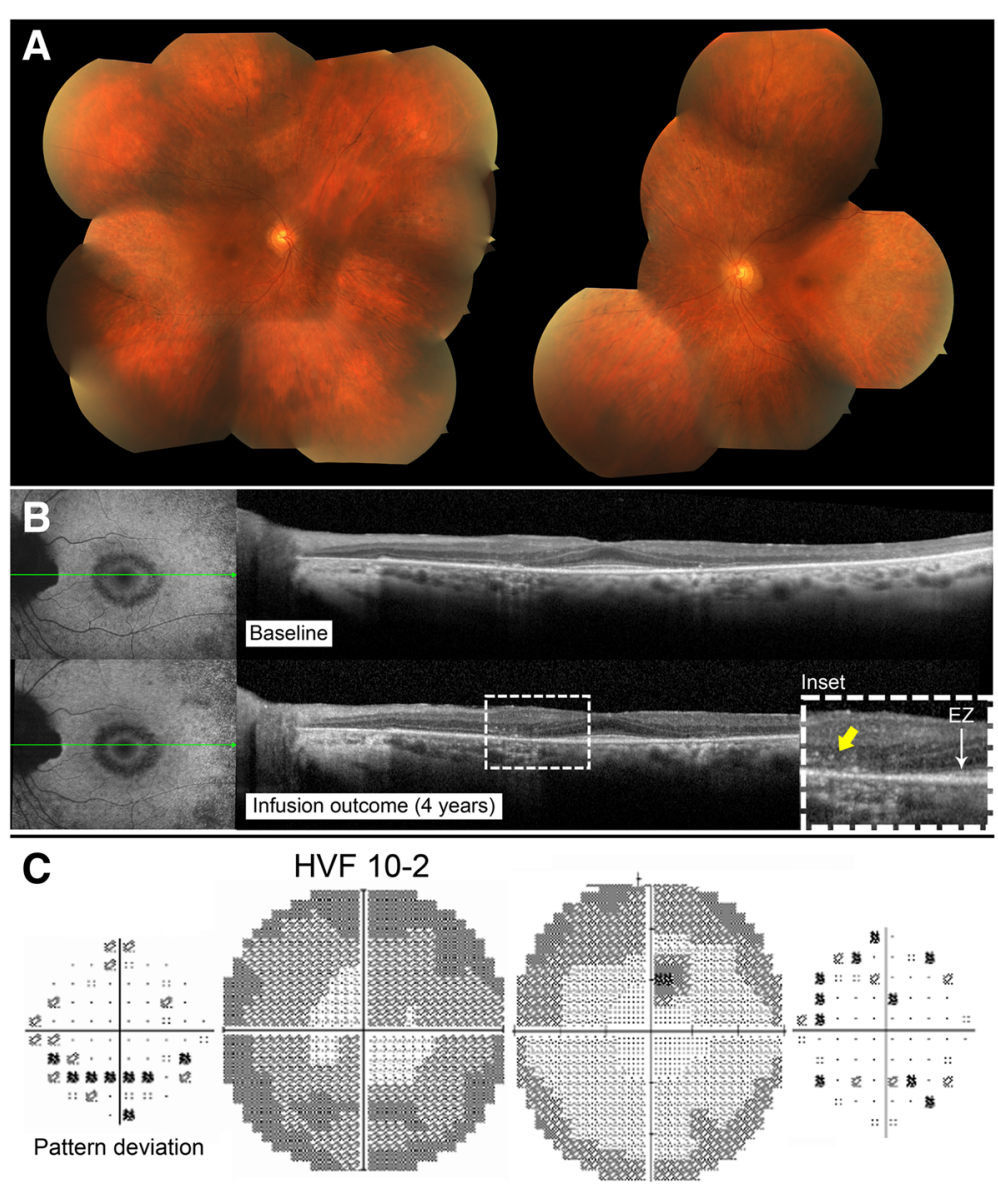

Fig. 7 P5 imaging and functional assessments. P5 fundus pictures OU (a). OCT line OD showing stability over many years when comparing OCT at presentation $(2011$, top) and after rituximab initiation $(2015$, bottom) (b). Humphrey visual field 24-2 grey scale and pattern deviation OD before (2014, left) and after (2015, right) initiation of rituximab showing mild improvement on overall sensitivity (c)

although the beneficial effects eventually subsided. A recent study reported that in a subgroup of nPAIR patients, roughly $63 \%$ responded well to immunosuppressive drugs such as cyclosporine, mycophenolate mofetil, infliximab, IVIg or steroids [27]. Although this estimate is higher than what was observed in other studies [6], patients' variable and transient response to immunosuppression is not surprising given the incertitude in the pathophysiology of this disease, including the questionable pathogenicity of many subtypes of ARAs to the retina $[28,29]$. Frequent failure of treatment has encouraged the pursuit of alternative drug strategies, rituximab being one example.

Rituximab is an immunosuppressant that has been used more recently in a number of systemic conditions, including patients with IgG4-related orbital diseases [30], myasthenia Gravis [31], neuromyelitis optica [32], and other ocular inflammatory or autoimmune conditions [33]. Binding of the drug to cell receptors leads to a rapid depletion in the population of $\mathrm{B}$ cells for approximately 6-12 months [34]. It is hypothesized that rituximab induces B-cell apoptosis through the activation of mitogen-activated protein kinases, natural killer cells, or the complement cascade [11].

In this case series, we found variable responses among five npAIR patients taking rituximab. Two appeared to stabilize, while one marginally improved and two others did not respond. Although treatment regimen varied between patients, all of them reached adequately low levels of B cells, confirming the potency of the drug. There are several possible reasons why treatment outcomes were inconsistent among patients. As mentioned, four patients had previously been prescribed immunosuppressants, and while three responded initially, they eventually became resistant to treatment. We hypothesize that improvement on rituximab may have been hampered by the limited number of functional photoreceptors or connected secondary-order cells remaining in the retinas of these patients. Additionally, each patient's genetic and immunological backgrounds may confer greater or lesser amenability of immunotherapy (including to rituximab) for the treatment of their condition, as has been suggested 
for other immunologically based retinal diseases [35]. In fact, some studies explain the low sensitivity to rituximab by citing differences in B cell memory capacity for reconfiguration [36] or the lack of specific complement regulatory proteins on the cell's surface [37] due to genetic differences among patients.

In addition, we did not observe a correlation between outcomes and changes in the type or level of antibodies after treatment in four out of four tested patients, which is unexpected. Indeed, in the two patients declared stable on rituximab, types (and level for one patient) of antibodies were similar at 5 and 8 months after starting rituximab. This might be because the CD20 receptor is not present on antibody-producing plasma cells, and thus, levels of immunoglobulin are not expected to decrease after rituximab infusion [34]. This phenomenon was observed in other studies as well. Looney et al. [38] reported improvement in patients with Lupus erythematosous following rituximab without changes in antidouble stranded DNA antibody or complement level. While Jarius et al. reported decreased antibody titers after rituximab treatment in neuromyelitis optica, the antibody always remained detectable in almost all patients [39]. Perhaps stability following treatment without change in antibody levels may be due to decreased antigen presentation rather than antibody level, [40] i.e. rituximab may be acting not only on B cell depletion, but on T-cell action as well [11]. Additionally, in P1, ARAs were still measurable after 7 months on rituximab but became undetectable 14 months later. Further studies are indicated to identify the appropriate time interval for repeat testing after baseline, although our data suggest that early on, antibody levels may not serve as a suitable proxy for patients' response to rituximab. Instead, this test may be best interpreted in conjunction with other indices of retinal function and structure (BCVA, ffERG, multi-modal imaging, etc.).

ERG is a relatively objective test that not only serves as an important tool in diagnosing AIR, but also enables assessment of the severity of retinal dysfunction. Monitoring patients' response to treatment is challenging, as many visual tests such as BCVA and visual field exams are subjective and vary based on the patient's affect, learning curve for complex tests, and cooperation [41-43]. For example, Mizener et al. observed that ERG was more sensitive than visual fields in assessing progression of three patients with npAIR [2]. Despite some variability between sessions, ERG testing in this study also proved to be an effective tool for monitoring patients over time, and changes in ERG outcomes correlated closely with patient symptoms [44], thus highlighting the utility of this test.

ERG data on npAIR patients is not as abundant as it is for patients with CAR [45-47] and MAR [48]. In general, while some patients present with greater rod than cone dysfunction initially, for a small minority, cones are affected first [8]. In this case series, four out of five patients presented with moderate to severe rod-cone dysfunction on ffERG, while only one showed cone-rod dysfunction initially. An electronegative appearance on the maximal response was detected in P1 and P3, suggesting that the inner retinal layers were most affected. The variable effect of different ARAs in each patient may explain this disturbance pattern wherein the inner retinal layers are targeted, which has been demonstrated frequently in MAR but is less commonly reported in CAR [49] and npAIR [2]. With progression of the disease, the ERG recording eventually becomes extinguished in most patients. Our findings demonstrate that ERG is an effective strategy for monitoring npAIR patients over time in an objective manner that facilitates clinical decision making by supplementing findings from retinal imaging, BCVA, and visual field tests.

Thus far, two case reports have been published studying the response of patients with npAIR to rituximab, and in each $[14,16]$, patients were reported to benefit from the drug, with overall improvements in retinal function. Additionally, one case series studied six npAIR patients receiving rituximab and/or combination therapy [19]. They found that following mono- or combinatorial therapy, $66.7 \%$ of eyes had stable visual acuity, 50\% showed stability on visual field testing, and 33.3\% showed stability or improvement on ERG. They also found that at least one pathogenically proven ARA band resolved after treatment. Overall, they concluded that stability or improvement on two or more tests in $83.5 \%$ of patients could be considered a successful treatment. Contrastingly, we observed much more variability among patients in our cohort and concluded that only $60 \%$ were stabilized or improved following treatment.

There are several notable differences in study design that may account for the differences observed in our findings. The primary difference lies in the standardization of the dosage of rituximab, which was administered at $375 \mathrm{mg} / \mathrm{m}^{2}$ every week for 8 weeks, then $375 \mathrm{mg} / \mathrm{m}^{2}$ monthly [50,51]. Contrastingly, patients in our study were treated with rituximab in a customized fashion based on symptoms and specialist preferences. A secondary difference is their combinatorial approach, wherein rituximab was co-administered with oral cyclophosphamide or bortezomib in 4 out of 6 of their patients, while ours were treated with rituximab exclusively. However, there are many points of consensus between our studies: visual acuity was on average stabilized in both cohorts, adverse events occurred in a minority of patients, and we both found unpredictable results in ARA outcomes, making their interpretation challenging. Additionally, our findings build on Foster et al.'s by supplying OCT and ARA titer testing results, 
although repeated testing among larger cohorts is still greatly needed.

Some limitations of our study should be acknowledged. While one of our patients was under 10 years of age and one older than sixty, the typical npAIR diagnosis is made between the ages of 20 and 25 years of age. Additionally, the regiment of rituximab was not standardized across patients. Some patients followed the rituximab protocol that was developed for B-cell lymphoma, while some were prescribed a regiment that was originally designed for rheumatoid arthritis patients, and yet others used non-standard protocols. Additional experience with other patients with autoimmune disorders and retinal atrophies may guide dosing of rituximab in the future. Additionally, the time point of assessment and antibody testing following rituximab infusions were also different for each patient, and the optimal follow-up time after infusion cannot be determined from these data. Testing blood before and at varying intervals after each rituximab infusion is one strategy that could determine the ideal timing for ARA testing in future studies, which in turn may enable better delineation of the drug's effects on ARAs. Finally, ratio analysis for ERG was used in this series in order to obtain an efficient comparison strategy of responses at different points for each patient, although actual voltage numbers could also have been used.

\section{Conclusions}

Overall, our findings suggest that rituximab may stabilize the progression of retinal dysfunction in some patients with advanced npAIR, although expectations for improvement should be temperate. The recommended interval for ARA testing following rituximab administration, as well as the use of this measure to drive decision making in isolation from other tests, remains an important consideration for future studies. To obtain an approximation of patients' progression and response to treatment, ffERG $30 \mathrm{~Hz}$-flicker can be used alongside other assessments, like ARA measures, to obtain a complete overview of patients' progression and response to treatment. Several patients initially responded well to other immunosuppressive therapies, suggesting that treatment should be readily considered rather than mere observation of the patient. In future studies, it would be valuable to explore whether earlier administration after diagnosis may enable greater improvements in patient outcomes.

\footnotetext{
Abbreviations

AIR: Autoimmune retinopathy; ARAs: Antiretinal antibodies; B-cells: B lymphocytes; BCVA: Best corrected visual acuity; CAR: Cancer-associated retinopathy; ERG: Electroretinogram; EZ: Ellipsoid zone; ffERGs: Full-field electroretinograms; HVF: Humphrey visual field; ISCEV: International Society for Clinical Electrophysiology of Vision; IVlg: Intravenous immunoglobulins; MAR: Melanoma-associated retinopathy; NCL: Neuronal ceroid lipofuscinose npAIR: Non-paraneoplastic autoimmune retinopathy; NYPH: New York
}

Presbyterian Hospital; OD: Right eye; OS: Left eye; pAIR: Paraneoplastic autoimmune retinopathy; RPE: Retinal pigment epithelium; SD-OCT: Spectraldomain optical coherence tomography; $\mu \mathrm{V}$ : Microvolts

\section{Acknowledgements \\ Funding \\ The Jonas Children's Vision Care and the Bernard \& Shirlee Brown Glaucoma Laboratory are supported by the National Institutes of Health [5P30EY019007, R01EY018213, R01EY024698, R01EY026682, R21AG050437], National Cancer Institute Core [5P30CA013696], the Research to Prevent Blindness (RPB) Physician-Scientist Award, unrestricted funds from RPB, New York, NY, USA. J.D.S. is supported by the RPB medical student fellowship. T.C. is supported by the International Council of Ophthalmology - Retina Research Foundation Helmerich Fellowship, honoring Mr. W. H. Helmerich III. S.H.T. is a member of the RD-CURE Consortium and is supported by the Tistou and Charlotte Kerstan Foundation, the Schneeweiss Stem Cell Fund, New York State [C029572], the Foundation Fighting Blindness New York Regional Research Center Grant [C-NY05-0705-0312], the Crowley Family Fund, and the Gebroe Family Foundation.}

\section{Availability of data and materials}

The datasets used and/or analyzed for the current study are available from the corresponding author upon reasonable request.

\section{Authors' contributions}

$\mathrm{KB}$ analyzed and interpreted the patient data regarding the retinal disease and electrophysiological response to treatment. SJ helped collect the data and was a major contributor in writing the manuscript. WL was a major contributor in editing the figures. All authors read and approved the final manuscript.

\section{Ethics approval and consent to participate}

This study was approved by the Institutional Review Board at Columbia University Medical Center (New York City) and complies with the Declaration of Helsinki. Written informed consent to participate was obtained from all subjects.

\section{Consent for publication}

Written informed consent for publication was obtained from all participants.

\section{Competing interests}

The authors declare that they have no competing interests.

\section{Publisher's Note}

Springer Nature remains neutral with regard to jurisdictional claims in published maps and institutional affiliations.

\section{Author details}

${ }^{1}$ Department of Ophthalmology, University of Montreal, Montreal, Canada. ${ }^{2} J o n a s$ Children's Vision Care, and Bernard \& Shirlee Brown Glaucoma Laboratory, Columbia University Medical Center, New York, NY, USA.

${ }^{3}$ Department of Ophthalmology, Columbia University, New York, NY, USA. ${ }^{4}$ State University of New York Downstate Medical Center, Brooklyn, NY, USA. ${ }^{5}$ Department of Ophthalmology, Federal University of Sao Paulo (UNIFESP), São Paulo, Brazil. ${ }^{6}$ Department of Ophthalmology, Federal University of Espírito Santo (UFES), Vitória, Brazil. Department of Pathology \& Cell Biology, Institute of Human Nutrition, College of Physicians and Surgeons, Columbia University, New York, NY, USA.

Received: 23 April 2017 Accepted: 5 July 2017

Published online: 15 July 2017

\section{References}

1. Sawyer RA, Selhorst JB, Zimmerman LE, Hoyt WF. Blindness caused by photoreceptor degeneration as a remote effect of cancer. Am J Ophthalmol. 1976;81:606-13.

2. Mizener JB, Kimura AE, Adamus G, Thirkill CE, Goeken JA, Kardon RH. Autoimmune retinopathy in the absence of cancer. Am J Ophthalmol. 1997;123:607-18. 
3. Heckenlively JR, Ferreyra HA, Jayasundera T. Controversies of diagnosing autoimmune retinopathy. Arch Ophthalmol. 2010;128:147-8. author reply 148-149

4. Braithwaite T, Vugler A, Tufail A. Autoimmune retinopathy. Ophthalmologica. 2012;228:131-42.

5. Keltner JL, Thirkill CE. Cancer-associated retinopathy vs recoverin-associated retinopathy. Am J Ophthalmol. 1998;126:296-302.

6. Weleber RG, Watzke RC, Shults WT, Trzupek KM, Heckenlively JR, Egan RA, Adamus G. Clinical and electrophysiologic characterization of paraneoplastic and autoimmune retinopathies associated with antienolase antibodies. Am J Ophthalmol. 2005;139:780-94

7. Adamus G, Brown L, Weleber RG. Molecular biomarkers for autoimmune retinopathies: significance of anti-transducin-alpha autoantibodies. Exp Mo Pathol. 2009;87:195-203.

8. Grewal DS, Fishman GA, Jampol LM. Autoimmune retinopathy and antiretinal antibodies: a review. Retina. 2014;34:827-45.

9. Fox AR, Gordon LK, Heckenlively JR, Davis JL, Goldstein DA, Lowder CY, Nussenblatt RB, Butler NJ, Dalal M, Jayasundera T, et al. Consensus on the diagnosis and Management of Nonparaneoplastic Autoimmune Retinopathy using a modified Delphi approach. Am J Ophthalmol. 2016;168: 183-90.

10. Nguyen DT, Amess JA, Doughty H, Hendry L, Diamond LW. IDEC-C2B8 antiCD20 (rituximab) immunotherapy in patients with low-grade non-Hodgkin's lymphoma and lymphoproliferative disorders: evaluation of response on 48 patients. Eur J Haematol. 1999;62:76-82.

11. Gurcan HM, Keskin DB, Stern JN, Nitzberg MA, Shekhani H, Ahmed AR. A review of the current use of rituximab in autoimmune diseases. Int Immunopharmacol. 2009;9:10-25.

12. Mahdi N, Faia LJ, Goodwin J, Nussenblatt RB, Sen HN. A case of autoimmune retinopathy associated with thyroid carcinoma. Ocul Immunol Inflamm. 2010;18:322-3.

13. Turaka K, Kietz D, Krishnamurti L, Mitchell E, Scanga H, Fu VL, Sylvester C. Carcinoma-associated retinopathy in a young teenager with immature teratoma of the ovary. J AAPOS. 2014;18:396-8.

14. Uludag G, Onal S, Arf S, Muslubas IS, Selcukbiricik F, Akbay AK, Mandel NM. Electroretinographic improvement after rituximab therapy in a patient with autoimmune retinopathy. Ajocasereports. 2016;2:4-7.

15. Or C, Collins DR, Merkur AB, Wang Y, Chan CC, Forooghian F. Intravenous rituximab for the treatment of cancer-associated retinopathy. Can J Ophthalmol. 2013;48:e35-8.

16. Fox A, Jeffrey B, Hasni $S$, Nussenblatt $R$, Sen HN. Rituximab treatment for nonparaneoplastic autoimmune retinopathy. Can J Ophthalmol. 2015;50:e101-4.

17. Dy I, Chintapatla R, Preeshagul I, Becker D. Treatment of cancer-associated retinopathy with rituximab. J Natl Compr Cancer Netw. 2013;11:1320-4.

18. Sen HN, Chan CC, Caruso RC, Fariss RN, Nussenblatt RB, Buggage RR. Waldenstrom's macroglobulinemia-associated retinopathy. Ophthalmology. 2004:111:535-9.

19. Maleki A, Lamba N, Ma L, Lee S, Schmidt A, Foster CS. Rituximab as a monotherapy or in combination therapy for the treatment of nonparaneoplastic autoimmune retinopathy. Clin Ophthalmol. 2017;11:377-85

20. Adamus G, Ren G, Weleber RG. Autoantibodies against retinal proteins in paraneoplastic and autoimmune retinopathy. BMC Ophthalmol. 2004;4:5.

21. Adamus G, Karren L. Autoimmunity against carbonic anhydrase II affects retinal cell functions in autoimmune retinopathy. J Autoimmun. 2009;32:133-9.

22. McCulloch DL, Marmor MF, Brigell MG, Hamilton R, Holder GE, Tzekov R, Bach M. ISCEV standard for full-field clinical electroretinography (2015 update). Doc Ophthalmol. 2015:130:1-12.

23. Craiu D, Dragostin O, Dica A, Hoffman-Zacharska D, Gos M, Bastian AE, Gherghiceanu M, Rolfs A, Nahavandi N, Craiu M, lliescu C. Rett-like onset in late-infantile neuronal ceroid lipofuscinosis (CLN7) caused by compound heterozygous mutation in the MFSD8 gene and review of the literature data on clinical onset signs. Eur J Paediatr Neurol. 2015;19:78-86.

24. Roosing S, van den Born LI, Sangermano R, Banfi S, Koenekoop RK, Zonneveld-Vrieling MN, Klaver CC, van Lith-Verhoeven JJ, Cremers FP, den Hollander Al, Hoyng CB. Mutations in MFSD8, encoding a lysosomal membrane protein, are associated with nonsyndromic autosomal recessive macular dystrophy. Ophthalmology. 2015;122:170-9.

25. Hardy RR, Hayakawa K. B cell development pathways. Annu Rev Immunol. 2001;19:595-621.

26. Berson EL. Long-term visual prognoses in patients with retinitis pigmentosa: the Ludwig von Sallmann lecture. Exp Eye Res. 2007:85:7-14.
27. Ferreyra HA, Jayasundera T, Khan NW, He S, Lu Y, Heckenlively JR. Management of autoimmune retinopathies with immunosuppression. Arch Ophthalmol. 2009;127:390-7.

28. Patel N, Ohbayashi M, Nugent AK, Ramchand K, Toda M, Chau KY, Bunce C, Webster A, Bird AC, Ono SJ, Chong V. Circulating anti-retinal antibodies as immune markers in age-related macular degeneration. Immunology. 2005;115:422-30.

29. Ahn BY, Song ES, Cho YJ, Kwon OW, Kim JK, Lee NG. Identification of an anti-aldolase autoantibody as a diagnostic marker for diabetic retinopathy by immunoproteomic analysis. Proteomics. 2006;6:1200-9.

30. Wu A, Andrew NH, Tsirbas A, Tan P, Gajdatsy A, Selva D. Rituximab for the treatment of lgG4-related orbital disease: experience from five cases. Eye (Lond). 2015;29:122-8.

31. Illa I, Diaz-Manera J, Rojas-Garcia R, Pradas J, Rey A, Blesa R, Juarez C, Gallardo E. Sustained response to Rituximab in anti-AChR and antiMuSK positive myasthenia gravis patients. J Neuroimmunol. 2008;201202:90-4

32. Zephir H, Bernard-Valnet R, Lebrun C, Outteryck O, Audoin B, Bourre B, Pittion S, Wiertlewski S, Ouallet JC, Neau JP, et al. Rituximab as first-line therapy in neuromyelitis optica: efficiency and tolerability. J Neurol. 2015:262:2329-35.

33. Miserocchi E, Pontikaki I, Modorati G, Gattinara M, Meroni PL, Gerloni V. Anti-CD 20 monoclonal antibody (rituximab) treatment for inflammatory ocular diseases. Autoimmun Rev. 2011;11:35-9.

34. Kosmidis ML, Dalakas MC. Practical considerations on the use of rituximab in autoimmune neurological disorders. Ther Adv Neurol Disord. 2010;3:93-105.

35. Gass JD. Are acute zonal occult outer retinopathy and the white spot syndromes (AZOOR complex) specific autoimmune diseases? Am J Ophthalmol. 2003:135:380-1.

36. Maurer MA, Rakocevic G, Leung CS, Quast I, Lukacisin M, Goebels N, Munz C, Wardemann H, Dalakas M, Lunemann JD. Rituximab induces sustained reduction of pathogenic $B$ cells in patients with peripheral nervous system autoimmunity. J Clin Invest. 2012;122:1393-402.

37. Hatjiharissi E, Xu L, Santos DD, Hunter ZR, Ciccarelli BT, Verselis S, Modica M, Cao Y, Manning RJ, Leleu X, et al. Increased natural killer cell expression of CD16, augmented binding and ADCC activity to rituximab among individuals expressing the Fc\{gamma\}RIIla-158 V/N and V/F polymorphism. Blood. 2007;110:2561-4.

38. Looney RJ, Anolik JH, Campbell D, Felgar RE, Young F, Arend LJ, Sloand JA, Rosenblatt J, Sanz I. B cell depletion as a novel treatment for systemic lupus erythematosus: a phase I/II dose-escalation trial of rituximab. Arthritis Rheum. 2004:50:2580-9.

39. Jarius S, Aboul-Enein F, Waters P, Kuenz B, Hauser A, Berger T, Lang W, Reindl M, Vincent A, Kristoferitsch W. Antibody to aquaporin-4 in the longterm course of neuromyelitis optica. Brain. 2008;131:3072-80.

40. Looney RJ. Treating human autoimmune disease by depleting B cells. Ann Rheum Dis. 2002;61:863-6.

41. Bittner AK, Iftikhar MH, Dagnelie G. Test-retest, within-visit variability of Goldmann visual fields in retinitis pigmentosa. Invest Ophthalmol Vis Sci. 2011;52:8042-6.

42. Kiser AK, Mladenovich D, Eshraghi F, Bourdeau D, Dagnelie G. Reliability and consistency of visual acuity and contrast sensitivity measures in advanced eye disease. Optom Vis Sci. 2005;82:946-54.

43. Ross DF, Fishman GA, Gilbert LD, Anderson RJ. Variability of visual field measurements in normal subjects and patients with retinitis pigmentosa. Arch Ophthalmol. 1984;102:1004-10.

44. Jampol LM, Fishman GA. Immunosuppression for autoimmune retinopathy. Arch Ophthalmol. 2009;127:573-5.

45. Klingele TG, Burde RM, Rappazzo JA, Isserman MJ, Burgess D, Kantor O. Paraneoplastic retinopathy. J Clin Neuroophthalmol. 1984;4:239-45.

46. Matsui Y, Mehta MC, Katsumi O, Brodie SE, Hirose T. Electrophysiological findings in paraneoplastic retinopathy. Graefes Arch Clin Exp Ophthalmol. 1992;230:324-8.

47. Thirkill CE, Keltner JL, Tyler NK, Roth AM. Antibody reactions with retina and cancer-associated antigens in 10 patients with cancer-associated retinopathy. Arch Ophthalmol. 1993;111:931-7.

48. Keltner JL, Thirkill CE, Yip PT. Clinical and immunologic characteristics of melanoma-associated retinopathy syndrome: eleven new cases and a review of 51 previously published cases. J Neuroophthalmol. 2001;21: 173-87. 
49. Anastasakis A, Dick AD, Damato EM, Spry PG, Majid MA. Cancer-associated retinopathy presenting as retinal vasculitis with a negative ERG suggestive of on-bipolar cell pathway dysfunction. Doc Ophthalmol. 2011;123:59-63.

50. Cao JH, Oray M, Cocho L, Foster CS. Rituximab in the treatment of refractory noninfectious Scleritis. Am J Ophthalmol. 2016;164:22-8.

51. Foster CS, Chang PY, Ahmed AR. Combination of rituximab and intravenous immunoglobulin for recalcitrant ocular cicatricial pemphigoid: a preliminary report. Ophthalmology. 2010;117:861-9.

Submit your next manuscript to BioMed Central and we will help you at every step:

- We accept pre-submission inquiries

- Our selector tool helps you to find the most relevant journal

- We provide round the clock customer support

- Convenient online submission

- Thorough peer review

- Inclusion in PubMed and all major indexing services

- Maximum visibility for your research

Submit your manuscript at www.biomedcentral.com/submit 